Supporting Information

\title{
Controllable Multicompartmental Capsules with Distinct Cores and Shells for Synergistic Release
}

Fan He, ${ }^{\dagger}$ Wei Wang, ${ }^{*}{ }^{\dagger}$ Xiao-Heng He, ${ }^{\dagger}$ Xiu-Lan Yang, ${ }^{\dagger}$ Ming Li, ${ }^{\dagger}$ Rui Xie, ${ }^{\dagger}$ Xiao-Jie Ju, ${ }^{\dagger}$ Zhuang Liu, ${ }^{\dagger}$ and Liang-Yin Chu ${ }^{*+, t, \xi}$

$+$

School of Chemical Engineering, Sichuan University, No. 24, Southern 1 Section, Yihuan Road, Chengdu, Sichuan 610065, P. R. China

$\ddagger$

State Key Laboratory of Polymer Materials Engineering, Sichuan University, Chengdu, Sichuan 610065, P. R. China

$\S$

Jiangsu National Synergetic Innovation Center for Advanced Materials (SICAM), Nanjing, Jiangsu 211816, P. R. China

*Corresponding Authors. E-mail: chuly@scu.edu.cn (L.Y.C.), wangwei512@ scu.edu.cn (W.W.) 


\section{Supplementary Figure}

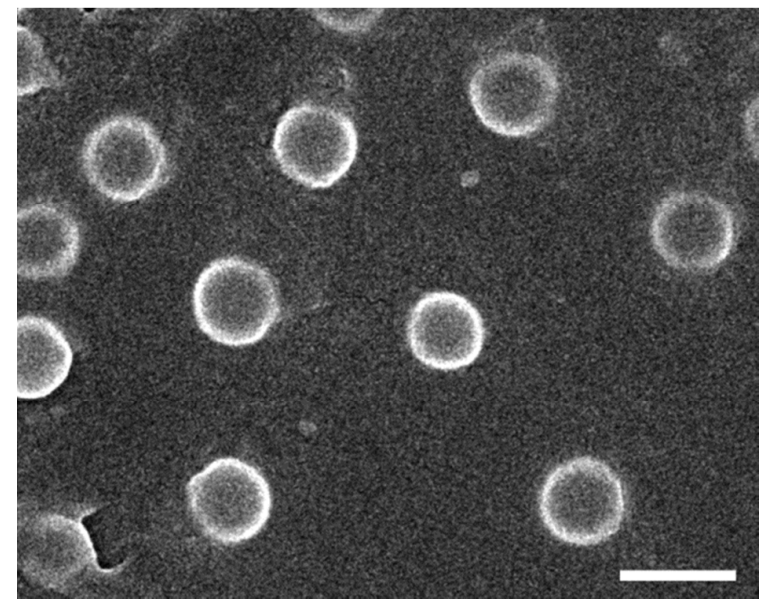

Figure S1. FESEM image of air-dried PNIPAM nanogels. The scale bar is $500 \mathrm{~nm}$. 


\section{Captions for Supplementary Movies}

Supplementary Movie S1. Coalescence process of two W/W droplets triggered by a stainless steel needle for fabricating dual-compartmental capsules.

Supplementary Movie S2. Magnetic-guided rotational motion of a dual-compartmental capsule under rotational magnetic field.

Supplementary Movie S3. Magnetic-guided translational movement of the dual-compartmental capsules directed by a magnet. 